\title{
Filigrane
}

Écoutes psychothérapiques

\section{Du rêve dans les romans de Philippe Sollers}

\section{Clara Ness}

Volume 16, numéro 2, automne 2007

Les hauts lieux et non-lieux du rêve II

URI : https://id.erudit.org/iderudit/016921ar

DOI : https://doi.org/10.7202/016921ar

Aller au sommaire du numéro

Éditeur(s)

Revue Santé mentale au Québec

ISSN

1192-1412 (imprimé)

1911-4656 (numérique)

Découvrir la revue

Citer cet article

Ness, C. (2007). Du rêve dans les romans de Philippe Sollers. Filigrane, 16(2), 54-61. https://doi.org/10.7202/016921ar

\section{Résumé de l'article}

D'Une curieuse solitude publié en 1958 au Seuil à Une vie divine paru en 2006 chez Gallimard, Clara Ness propose une analyse psychanalytique du rêve dans les romans de Philippe Sollers. S'ils se présentent comme des chants amoureux où est saluée la chance d'exister, les romans sollersiens prennent leur source sur fond tragique. Placé en incipit, le rêve offre un terrain privilégié pour témoigner de ce côté obscur, voire morbide, de l'intelligence. Le but du héros ? Se tirer de cet « enfer " pour accéder à la lumière, à une certaine forme de "Paradis". 


\title{
Du rêve dans les romans de Philippe Sollers ${ }^{1}$
}

\author{
clara ness
}

D'Une curieuse solitude publié en 1958 au Seuil à Une vie divine paru en 2006 chez Gallimard, Clara Ness propose une analyse psychanalytique du rêve dans les romans de Philippe Sollers. S'ils se présentent comme des chants amoureux où est saluée la chance d'exister, les romans sollersiens prennent leur source sur fond tragique. Placé en incipit, le rêve offre un terrain privilégié pour témoigner de ce côté obscur, voire morbide, de l'intelligence. Le but du héros? Se tirer de cet «enfer» pour accéder à la lumière, à une certaine forme de «Paradis».

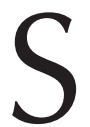

i les romans de Philippe Sollers se présentent comme des chants amoureux où est saluée la chance d'exister, thème honni par la plupart des écrivains contemporains, ils tiennent aussi compte de «l'enfer des femmes là-bas». Comment une écriture de la grâce, de la finesse pourrait-elle prendre autrement sa source que sur fond tragique? À quel prix cette résistance enchantée peut-elle s'acquérir? Comme si, seule la lente, la pénible ascension, de l'enfer au paradis, permettait au narrateur des romans Sollersiens de «disperser les nuées» et d'atteindre le «Salut », mot final, d'ailleurs, d'Une vie divine (2006). Le rêve, systématiquement placé en incipit de plusieurs de ses romans, est le témoin privilégié des méandres compliqués, souvent honteux, des pulsions inconscientes. Le but du héros ? Se tirer de cet «enfer» pour accéder à la lumière, à une certaine forme de «Paradis». En observant la majorité des romans de Sollers depuis Portrait du joueur (1984), on se rend compte que, par leurs schémas narratifs plus ou moins répétitifs (ascension, initiation ou libération du narrateur jusqu'au bonheur, associé chez Sollers à une forme d'ataraxie), ces romans parlent d'eux-mêmes et se regardent, s'exhibent et dévoilent leurs mécanismes en construction. Ainsi, la place initiale du rêve contribue à créer un motif récurrent: synonyme de mort, il n'est jamais regardé avec l'œil amusé des surréalistes ${ }^{2}$ ou la lorgnette voyeuse des psychanalystes. Chez Sollers, les rêves se placent du côté obscur, voire morbide, de l'intelligence: «Ça » parle, puisque «Nous parlons éveillés; nous parlons en rêve » (Heiddeger, 1976). Il y a, chez Sollers, une fascination mêlée de répulsion pour la question du rêve; ce bonheur de courte durée reste le lieu privilégié de l'intrusion du pulsionnel où se meuvent toutes les pensées traumatiques. Le rêve offre à la cisaille de l'écriture un formidable matériau brut à travailler. Loin de nous, donc, l'ésotérisme lié aux clés des songes, sauf si ce n'est, à l'exception du roman Le lys d'or (1989), le rêve d'un roman qui est en train de s'écrire sous nos yeux. Il s'agira non pas de se passionner pour ses pulsions mortifères mais au contraire, «comme disent les vieux textes, [...] ouvrir le crâne, élever le chant» (Sollers, 2002). 
Prenons un roman comme Portrait du joueur. Le narrateur, un écrivain ayant subi les critiques, les menaces, les âneries, les conversations de lectrices et de lecteurs outrés par la publication de son dernier roman (on imagine la part autobiographique de cet épisode, Sollers sortant à peine des remous qu'avait causé son roman Femmes, pense à se «tuer ici». «Me tuer ici ? Pourquoi pas ? Je n'aurais qu'à brûler tous les documents, traverser les vignes, entrer dans la forêt, avancer entre les pins, m'enfoncer, aller jusqu' au petit ruisseau noir, en bas, celui des écrevisses, avaler mes somnifères et, au moment de m'endormir à fond, appuyer sur la gâchette... Basta! Cervelle dans les sous-bois !» (Sollers, 1984). En d'autres termes, un véritable cauchemar éveillé, jusqu'à ce que le stylo, et les femmes, surtout une, puis deux, puis plusieurs autres viennent accentuer, puis apaiser ce dégoût. Il faut dire que le vocabulaire nocturne de Philippe Sollers s'accompagne systématiquement de connotations péjoratives ou mélioratives selon les cas; distinguons, dans un premier temps, «entrée dans le sommeil», «rêves» et «sommeil». "S'endormir» correspond la plupart du temps à un moment de grande angoisse ; l'état de passivité que nécessite le sommeil (relâchement de tous les muscles, vagabondage de la pensée) est presque toujours vécu comme l'élément déclencheur d'une crise physique ou intellectuelle. Dans Le coeur absolu (1987), au moment de se mettre au lit, le narrateur est secoué d'une crise d'épilepsie: «En m'endormant, ce soir-là, j'ai commencé à voir des fleurs entre mes yeux et l'obscurité me frise un peu au-dessus de ma tête, au-dessus du lit, dans le noir. [...] Ce serait drôle si j'étais mort, me suis-je dit un moment, mort avec des yeux en retard sur la fin de tout, allongé là, dans le fossé, dans la fosse, avec, comme couronne, cette haie rouge et blanche. » (Sollers, 1987). Même scène dans Passion fixe (2000) où le narrateur, aux prises avec un révolver qui tient lieu de choix philosophique vraiment sérieux (Camus), se demande s'il se suicidera ou non : «Le lit s'est mis lentement à s'abaisser au-dessous du lit, les murs à glisser sur eux-mêmes. Le lieu s'enfonçait et s'ouvrait à la fois, embarquement, enterrement fluide. » (Sollers 2000). Le corps, en position couchée, vulnérable à l'intrusion des pensées inconscientes et donc, des rêves, subit ici comme un avant-goût troublant de la mort.

Le «sommeil» est considéré quant à lui comme le lieu privilégié pour les amants de se protéger du monde extérieur. Sollers écrit, dans L'étoile des amants (2002): «Merci d'être là pendant ce temps-là, toi. On peut se balader en pleine grâce, en pleine laideur, on traverse, on oublie ce qu'il faut, on dort. » Ou encore : «On va dormir beaucoup, c'est nécessaire. Dormir et encore dormir, c'est la meilleure façon de leur échapper, et le plus possible d'un sommeil sans rêve », ce même «leur» renvoyant de façon détournée à tous les «anti-littérature » contre lesquels se battait le narrateur paranoïaque de Portrait du joueur; ces même «ils», responsables des bribes «chuchotées, martelées, conneries, éclats, obscénités, refus, reproches » (L'étoile des amants, 2000). Ainsi le sommeil, une fois que le corps et l'esprit y sont entièrement plongés, est nécessairement ressenti comme un état agréable: protégés du monde extérieur, les amants 
retrouvent un sentiment de béatitude, cet état rassurant de «narcissisme primaire» (Freud).

Regardons de plus près la question du rêve. Pour l'auteur du Cóur absolu, le rêve ne peut être témoin d'aucune vérité consciente, mais plutôt l'inverse, il est source de brouillage; ce sont les nuages obscurs de la pensée. On sait l'importance que Philippe Sollers accorde au XVIII ${ }^{\mathrm{e}}$ siècle et aux Lumières, et son horreur pour le retour de l'obscurantisme qui, à son avis, aurait recommencé au lendemain de la Révolution. L'important étant de faire les choses consciemment; tout abandon de la pensée serait considéré comme une nouvelle plongée en enfer. Dans L'étoile des amants, il l'écrit en ces termes: «...si on atteint la délivrance, c'est-à-dire le grand bonheur, on se retrouve dans un isolement admirable. [...] Comment ça vient? En marchant, en respirant, en restant assis, en respirant, en mangeant, en dormant, en faisant l'amour consciemment.» (L'étoile des amants, 2000). Mais justement, comment dormir consciemment? Voilà le cœur de l'angoisse: par glissement, si le rêve est « la voie royale de l'inconscient » (Freud), il est humiliant pour le sujet de s'avouer que la part consciente de l'esprit n'est pas maître à bord. On conçoit l'ampleur du problème: la veille «consciente» est survalorisée puisque quand «c'est conscient, c'est heureux » (L'étoile des amants, 2000) tandis que l'entrée dans le sommeil, accompagnée d'une incontournable angoisse de castration, s'associe à un repli sur soi, à une obsession de la mort, à la découverte chaque nuit renouvelée de ce terreau fertile des névroses.

À moins que les rêves ne soient l'objet du roman lui-même; dans ce cas ils seront révélateurs d'un livre en soi. C'est le cas avec Le lys d'or. Simon Rouvray, professeur de chinois au Centre d'études religieuses, rencontre une richissime héritière de vingt-huit ans, Reine de Laume. Elle va lui demander d'écrire, à ses frais, sans rien cacher, le récit de sa vie et de leurs relations ambiguës. En 1989, après le Nouveau Roman, après l'époque Tel Quel, Le lys d'or renoue avec les vieux démons qui avaient fait les belles années de la théorie littéraire française: à nouveau la littérature parlera de littérature, elle se questionnera sur son fonctionnement. Comme un gant qui se retourne, ses mécanismes seront entièrement exhibés. On pense aux premiers romans de Sollers, Nombres, Lois, $H$ et surtout Paradis, ou encore à la «Déclaration» dans le premier numéro de Tel Quel: «Ce qu'il faut dire aujourd'hui, c'est que l'écriture n'est plus concevable sans une claire prévision de ses pouvoirs, un sang-froid à la mesure du chaos où elle s'éveille, une détermination qui mettra la poésie à la plus haute place de l'esprit. » («Déclaration», 1960.)

La littérature, devenue «expérience à vivre», selon le mot de Michaux, ne cachera plus ses procédés; dévoilant ses rouages, l'œuvre littéraire se tourne vers elle-même et regarde «ce phénomène du langage qu'au plus profond d'elle-même elle constitue dans le moment même où se développe la certitude que le langage est en soi la dimension constitutive de toute conscience humaine » (Forest, 1995). C'est ce qui se produira dans Le lys d'or et dans Une vie divine, où la place du rêve aura un rôle de personnage à part entière dans la construction du récit. 
Le lys d'or commence ainsi: «Premier rêve: je suis debout dans l'herbe, à genoux, la tempête bat son plein, marée haute gris-vert agité du ciel et de l'eau, je continue à creuser, je m'écorche les doigts, je saigne. [...] Je dois absolument retrouver ce paquet enterré l'année dernière, je revois la double housse de plastique bleu, c'était bien là, à droite du laurier, pas très profond, personne n'a pu deviner l'endroit...» (Sollers 1989). Puis: «Deuxième rêve: je suis dans la chambre avec Marie. Quelque chose de terrible est arrivé à Paul, il est sans doute tombé dans l'escalier, son petit corps recroquevillé est là, sous les draps. [...] C'est le grand beau temps de la mort. » (Le lys d'or, 2000). On retrouve l'angoisse de castration dans le premier rêve («je m'écorche les doigts, je saigne») de même qu'un motif récurrent des incipit sollersiens : le narrateur doit retrouver un objet, la plupart du temps un carnet de notes, ou un journal, abandonné l'année précédente, caché dans un tiroir ou dans le sol. L'important est qu'il ne le retrouve pas. Souvent, il s'agit d'un récit en cours, ou encore de notes plus précises sur une situation vécue comme dans Une vie divine:

\section{$\ll$ - Et le cahier?}

— En haut, dans le tiroir du bureau, à droite.

Je me suis entendu répondre ça, un réflexe. En réalité, je ne pensais plus du tout à ce cahier d'il y a dix ans, des notes sur mon expérience.»

Dans Le lys d'or, ce premier rêve préfigure le roman à venir : Simon Rouvray, futur biographe de (la) Reine, creuse le sol à la recherche d'une idée. Reine arrive trois pages plus loin; il recopie l'incipit et écrit «Le titre en sera: LE LYS D'OR» (2000). Autre exemple: Le lys d'or est un roman en six parties. Vers la toute fin du roman, le narrateur s'explique comme suit à propos de cette construction: «Six pétales, six étamines, un pistil. [...] Je vous écris ce truc en six parties, vous voyez, pour des raisons profondes.» (Le lys d'or, 2000). Ce dispositif de redoublement narratif sera le véritable sujet de ce roman fleuri. Il faut analyser le deuxième rêve pour mieux comprendre les raisons de ce télescopage de narrations. Simon Rouvray rêve à son enfant mort. On devine la culpabilité des parents face à un tel désir refoulé. De plus, «son petit corps est recroquevillé là, sous les draps », de façon, peut-être, à ce que les parents ne s'unissent plus. «C'est le grand beau temps de la mort ${ }^{3} »$ : ambivalence évidemment coupable à l'égard de cet insupportable souhait. Le processus de redoublement narratif éloigne le «je» de ce rêve scandaleux; Simon Rouvray délègue le récit de son livre à l'histoire de la vie de quelqu'un d'autre. Le lecteur est constamment balancé entre trois niveaux de lectures : d'une part, le récit, brut, de la vie de Reine; d'autre part, le professeur Simon Rouvray qui écrit une biographie qui deviendra par la suite un roman en soi, Le lys d'or. Il n'est donc pas étonnant qu'il y ait dès le début du récit deux rêves traumatisants : un premier, rempli d'une promesse non tenue, et un deuxième qui soit un désir honteux. Célébration de la «jouissance d'exister» (L'étoile des 
amants, 2002) sur fond fatidique: la peur des révélations scandaleuses de l'inconscient, qui prend l'ensemble de la pensée au moment des rêves, est une porte ouverte vers des désirs potentiellement traumatiques. Afin de rendre supportables ces rêves scandaleux, le narrateur se décharge constamment de sa responsabilité en se consacrant au récit de quelqu'un d'autre. Et comme par magie, dès l'instant où ces rêves sont révélés, l'angoisse des premières pages se dissipe. Le lys d'or amorce un nouveau cycle de romans où l'esprit de vengeance qui avait fait le sel de Femmes et de Portrait du joueur (où la mort frôlait de près la majorité des personnages à commencer par le narrateur) laisse place à une période d'apaisement, d'où naîtront des romans comme Passion fixe et L'étoile des amants.

Le topos du rêve reviendra dans le dernier livre de Sollers paru à ce jour, Une vie divine (2006). Un discours très précis sur le rêve se dévoile dès les premières pages du roman: "C'était un rêve, le vent, la dépression, le blocage, le cahier, Ludi. Ils sont là pour vous avertir, les rêves. Le tragique y est comique, le comique pathétique, la vie inguérissable, la mort au coin de la rue, le corps une hypothèse, l'identité une longueur d'onde en cours de brouillage.» (Sollers, 2006) Au début de ce roman, le narrateur, philosophe, se voit dans la glace de la salle de bain, tête ouverte, ayant perdu sa calotte, le cerveau à l'air. Ce rêve reviendra tout au long du récit, hommage à Hamlet bien sûr, au crâne dans le cimetière, ainsi qu'aux crânes peints par Cézanne ou sculptés par Picasso.

Il est évident que, pour l'auteur de Femmes, la pensée est investie de façon phallique; on tirera les conclusions de cette circoncision des fontanelles. Le corps est coupé de façon radicale au niveau de la tête, organe le plus vital avec le cœur. Non seulement il s'agit d'une angoisse de castration, mais aussi d'intrusion (angoisse féminine). On ne peut négliger la dimension exhibitionniste de ce crâne ouvert, toujours avec l'équivalence symbolique cerveau (intelligence) = pénis. Il y a dans l'écriture de Sollers un désir/angoisse de s'exhiber via les mots et l'écriture mais aussi qui, inconsciemment, renvoie/correspond à un désir d'exhibition sexuelle. On retrouve également la peur de la passivité (féminité) puisque l'exhibition c'est «se faire voir», c'est-à-dire éprouver le plaisir d'être en position passive face aux regards, même si cela est activement mis en œuvre. Un écrivain est en permanence aux prises avec ce genre de questionnement, surtout lorsqu'il est célèbre (le fait d'écrire sur soi, est-ce un geste exhibitionniste? Comment protéger les siens? etc). Finalement, un crâne ouvert, c'est aussi la peur inhérente que son contenu se vide, position gênante pour un écrivain, on en conviendra. On arrive au nœud de l'entreprise : est-ce que l'écriture du rêve n'est pas une manière de maitriser quelque chose de traumatique? Est-ce que la sublimation de l'écriture, comme toute entreprise d'intellectualisation, permet de mettre à distance, comme un ultime mécanisme de défense, les idées inconscientes supportables?

Il y a toujours une forme de réconciliation avec l'inconscient dans les romans sollersiens. Par exemple, dans Une vie divine, dans les moments de doute, le motif du «crâne» résonne comme un avertissement: «Je reprends mon crâne et ma forme habituelle, et, en effet, c'est moi. » (Une vie divine, 2006) Le rêve occupe la 
place d'un révélateur, d'un miroir, ou encore d'un baromètre de certitude: «Quelqu'un, en tout cas, qui a tendance à dire nous à ma place, c'est bien mon crâne, vu de nouveau en rêve dans le miroir. Il est là, irréfutable, neutre, collectif, vaguement comique, il me regarde depuis mon au-delà fatal» (Une vie divine, 2006) puis à la toute fin du roman: «Je me réveille, je m'endors, je continue à rêver en étant conscient que je rêve, je me réveille à nouveau [...]. C'est passé. Le jour est là, je me lève, je pose mon crâne, encore une fois, devant moi.» (Une vie divine, 2006). Le crâne (la mort, donc; on pense à Artaud, «La société se croit seule, mais il y a quelqu'un », comme si la mort butait sur cela: quelqu'un, symbole de la vanité des vanités) ramène le narrateur à sa finitude mortelle.

En d'autres mots, le rêve est admissible, avouable, chez Sollers, que s'il est conscient, c'est-à-dire ramené à l'état de langage. Cette ressource littéraire fait écho à une entreprise philosophique plus vaste ; on ne compte plus les publications de Sollers où les phrases de Hölderlin, «Là où le péril croît crô̂t aussi ce qui sauve » ou de Pindare, «L'homme est le rêve d'une ombre, mais quand les dieux dirigent sur lui un rayon, un éclat brillant l'environne, et son existence est douce», sont citées. Si, comme le dit Shakespeare, nous sommes faits «de l'étoffe dont sont faits les rêves », le travail d'écriture et de sublimation permet de dépasser l'angoisse de mort inhérente au sommeil.

Lors d'une série d'entretiens avec François Meyronnis et Yannick Haenel pour la revue Ligne de risque, Sollers explique ses prises de position philosophiques à ce sujet, amorcées dans des recueils comme La guerre du goût et Éloge de l'infini. Évoquant tantôt Rimbaud, tantôt Heidegger, il reproche à la littérature actuelle de ne s'intéresser qu'à la mort, d'où l'obsession de Sollers à en montrer la beauté: «Différents états permettent de faire l'expérience du néant. On a beaucoup mis en valeur — c'est le cas de le dire — l'ennui, l'angoisse. On a peu insisté sur la joie.» (Sollers, 2005). La littérature doit se concevoir comme un «dépassement de la métaphysique» (Poker, 2005); or, «Ce que la métaphysique ne tolère pas, c'est le fait qu'il y ait du destin [...] c'est-à-dire une passion fixe. Là, la haine ne connaît plus de bornes.» (Poker, 2005). L'obsession générale pour le ressentiment et l'esprit de vengeance peut être détournée par la littérature ${ }^{4}$, l'idée étant d'arriver à en «exécrer la misère (Une saison en enfer).

En d'autres termes, la mort n'est pas pour autant un tabou chez Sollers; les derniers mots de Portrait du joueur, roman qui rend hommage à l'aristocratie, aux grands vins du bordelais, aux courses de chevaux et à l'érotisme délicat de «Sophie», sont un cas unique: un rêve heureux est placé à la fin du récit. Métaphore subtile de la mort, elle est vécue sereinement: «Est-ce que le raisin ne va pas brûler? Non, il dort là, tranquillement, pressé, broyé, exalté, réglé. [...] Je referme les yeux, et je me vois tout à coup pousser mon attelage, là-bas, jusqu'au bout, vers l'ouest, [...], le rêve [des chevaux] se faufilent et foncent vers l'horizon rouge, $[\ldots]$ je lâche les rênes, ils veulent passer eux aussi, et peut-être vont-ils passer, malgré tout, museaux et naseaux comme directement vaporisés dans l'envers », «l'envers » étant cet aspect qui n'apparaît pas à première vue, qui n'est 
pas saisissable, palpable. Le travail d'écriture permet une ressaisie autrement plus sublime de cette part sombre de l'être humain. L'exergue du deuxième roman de Sollers, Une curieuse solitude (1958), tendait déjà vers cette volonté heureuse: «Le plus beau des courages, celui d'être heureux.» (Joubert). En rendant lumineuses les pensées obscures, il se positionne de façon radicalement opposée face à l'inconscient (active et non passive). Le dernier rêve cité, celui que nous appellerons des «chevaux», fait penser à cette phrase de Sigmund Freud dans Au-delà du principe de plaisir: «Le Moi ressemble dans sa relation au Ça au cavalier qui doit réfréner la force supérieure de son cheval.» (Freud, 1920). Dans ce rêve de Portrait du joueur, les chevaux «foncent vers l'horizon rouge» (le rouge, couleur symboliquement forte au niveau pulsionnel) et le narrateur «lâche les rênes »; en d'autres termes, une réconciliation avec l'inconscient est envisagée, la pulsion de vie est libérée.

Pour toutes ces raison, il y a donc un «Salut» possible, en-dehors du sens chrétien que ce mot évoque; pensons à Nietzsche: «Au-delà du nord, de la glace, de la mort - notre vie, notre bonheur... Nous avons découvert le bonheur, nous connaissons le chemin, nous avons trouvé l'issue de ces milliers d'années de labyrinthe». Est-ce vraiment un hasard si, de la part de celui qui s'était autoproclamé l'Antéchrist, cette phrase est citée en exergue d'Une vie divine? Supposons que non.

clara ness

7 , cours marigny 94300 vincennes france clara.ness@yahoo.fr

\section{Notes}

1. L'auteure tient à remercier, pour son aide à la rédaction de cet article, mademoiselle Fanny Dargent, psychologue.

2. En 1983, lorsqu'il publie Femmes, l'opinion de Sollers sur les surréalistes est assez ferme: «Le surréalisme! Parlons-en! Les pruderies occultes de Breton... L'imposture minaudière d'Aragon... L'exaltation anti-sexuelle d'Artaud... Seul Bataille garde un peu d'allure dans ce grand capharnaüm refoulant... Surtout par rapport à Sartre... La nausée... C'est le cas de le dire... Genet... Bref, sexuellement, rien... Quel désastre...».

3. Macabre coincidence: suite à la mort de son enfant, Philippe Forest, ami de Philippe Sollers, écrira L'enfant éternel, publié en 1997 chez Gallimard, où il écrira ceci à propos du rêve : «J'ai fait de ma fille un être de papier. J'ai tous les soirs transformé mon bureau en théâtre d'encre où se jouaient encore ses aventures inventées. Le point final est posé. J'ai rangé le livre avec les autres. Les mots ne sont plus d'aucun secours. Je fais ce rêve. Au matin, elle m'appelle de sa voix gaie au réveil. Je monte jusqu'à sa chambre. Elle est faible et souriante. Nous disons quelques mots ordinaires. Elle ne peut plus descendre seule l'escalier. Je la prends dans mes bras. Je soulève son corps infiniment léger. Sa main gauche s'accroche à mon épaule, elle glisse autour de moi son bras droit et dans le creux de mon cou je sens la présence tendre de sa tête nue. Me tenant à la rampe, la portant, je l'emmène avec moi. Et une fois encore, vers la vie, nous descendons les marches raides de l'escalier de bois rouge. »

4. Librement inspiré d'un entretien personnel avec Philippe Sollers réalisée le 15 février 2006. 


\section{Bibliographie}

Forest, Philippe, 1995, Histoire de Tel Quel 1960-1982, Paris, Seuil, coll. Fiction et Cie.

Forest, Philippe, 1997, L'enfant éternel, Paris, Gallimard.

Freud, Sigmund, 1920, Au-delà du principe de plaisir, Essais de psychanalyse, Payot, 2001.

«Déclaration», 1960, Tel Quel, n 1, Paris, Seuil, printemps.

HEeidegger, Martin, 1976, Acheminement vers la parole, Paris, Gallimard, coll. Tel.

Sollers, Phippe, 1958, Une curieuse solitude, Paris, Seuil

Sollers, Phippe, 1983, Femmes, Paris, Gallimard.

Sollers, Phippe, 1984, Portrait du joueur, Paris, Gallimard, Folio.

Sollers, Phippe, 1987, Le cœur Absolu, Paris, Gallimard, Folio.

Sollers, Phippe, 2000, Passion fixe, Paris, Gallimard.

Sollers, Phippe, 2002, L'étoile des amants, Paris, Gallimard.

Sollers, Phippe, 2005, Poker: Entretiens avec la revue Ligne de risque, Paris, Gallimard, coll. L'Infini.

Sollers, Phippe, 2006, Une vie divine, Paris, Gallimard. 